\title{
A Fast, Efficient and Easy to Implement Method to Purify Bacterial Pili From Lacticaseibacillus rhamnosus GG Based on Multimodal Chromatography
}

\author{
Raphael Dos Santos Morais ${ }^{*}$, Sofiane El-Kirat-Chatel', Jennifer Burgain', \\ Blandine Simard ${ }^{1}$, Sarah Barrau', Cédric Paris' ${ }^{1}$, Frédéric Borges ${ }^{1}$ and Claire Gaiani ${ }^{1,3 *}$ \\ ${ }^{1}$ Laboratoire d'Ingénierie des Biomolécules, Université de Lorraine, Nancy, France, ${ }^{2}$ Laboratoire de Chimie Physique et \\ Microbiologie pour les Matériaux et l'Environnement (LCPME), UMR 7564, CNRS-Université de Lorraine, Nancy, France, \\ ${ }^{3}$ Institut Universitaire de France, Parris, France
}

\section{OPEN ACCESS}

Edited by: Carlos Alberto Moreira-Filho, University of São Paulo, Brazil

Reviewed by:

Reetta Satokari,

University of Helsinki, Finland Rodrigo Dias De Oliveira Carvalho, Federal University of Minas Gerais,

Brazi

*Correspondence: Raphael Dos Santos Morais raphael.dos-santos-morais@ univ-lorraine.fr Claire Gaian

claire.gaiani@univ-lorraine.fr

Specialty section: This article was submitted to

Microbiotechnology,

a section of the journal

Frontiers in Microbiology

Received: 28 September 2020 Accepted: 24 November 2020 Published: 18 December 2020

Citation:

Dos Santos Morais R, El-Kirat-Chatel S, Burgain J,

Simard B, Barrau S, Paris C, Borges F and Gaiani C (2020) A Fast, Efficient and Easy to Implement Method to Purify Bacterial Pili From Lacticaseibacillus rhamnosus GG Based on Multimodal Chromatography.

Front. Microbiol. 11:609880 doi: 10.3389/fmich.2020.609880
Pili are polymeric proteins located at the cell surface of bacteria. These filamentous proteins play a pivotal role in bacterial adhesion with the surrounding environment. They are found both in Gram-negative and Gram-positive bacteria but differ in their structural organization. Purifying these high molecular weight proteins is challenging and has certainly slowed down their characterization. Here, we propose a chromatographybased protocol, mainly relying on multimodal chromatography (core bead technology using Capto Core 700 resin), to purify sortase-dependent SpaCBA pili from the probiotic strain Lacticaseibacillus rhamnosus GG (LGG). Contrary to previously published methods, this purification protocol does not require specific antibodies nor complex laboratory equipment, including for the multimodal chromatography step, and provides high degree of protein purity. No other proteins were detectable by SDS-PAGE and the $260 / 280 \mathrm{~nm}$ ratio ( $\sim$ 0.6) of the UV spectrum confirmed the absence of any other co-purified macromolecules. One can obtain $\sim 50 \mu \mathrm{g}$ of purified pili, starting from $1 \mathrm{~L}$ culture at $\mathrm{OD}_{600 \mathrm{~nm}} \approx 1$, in 2-3 working days. This simple protocol could be useful to numerous laboratories to purify pili from LGG easily. Therefore, the present work should boost specific studies dedicated to LGG SpaCBA pili and the characterization of the interactions occurring with their protein partners at the molecular level. Moreover, this straightforward purification process might be extended to the purification of sortase-dependant pili from other Gram-positive bacteria.

Keywords: Lacticaseibacillus rhamnosus GG, SpaCBA pili, purification, multimodal chromatography, light scattering, atomic force microscopy

\section{INTRODUCTION}

Pili, or fimbriae, are length-variable proteinaceous appendages found at the surface of bacteria and archaea where they play a key role in interaction and/or adhesion with the surrounding environment (Berne et al., 2018). These proteins exhibit a high structural organization diversity (Proft and Baker, 2008). Some types of pili are found in both Gram-negative and Gram-positive bacteria and archaea, such as type IV pili (Hospenthal et al., 2017; 
Wang et al., 2019). Other pili are exclusive to Gram-negative bacteria, such as chaperone usher, and others are specific to Gram-positive bacteria (Hospenthal et al., 2017). Indeed, Grampositive bacteria possess pili called sortase-dependent pili, named after the transmembrane enzyme catalyzing the reaction of polymerization. These pili are composed of three polymerized subunits, called pilin, covalently bound to each other, contrary to the other types of aforementioned pili. A single basal pilin allows for attachment to the cell wall, a major pilin is found in multiple copies which drives the length of the pilus, and a tip pilin located at the end of the pilus is responsible for interactions with the environment (Krishnan, 2015). Polymerization occurs through the reaction of a peculiar Lys of a pilin motif (exclusive to the basal and the major pilins) and a specific Thr found in the LPXTG motif which is common to the three pilins (Krishnan, 2015; von Ossowski, 2017). Remarkably, this kind of pili from Corynebacterium diphtheriae has successfully been rebuilt in vitro (Chang et al., 2018) and the reaction occurring between the pilin and the LPXTG motifs was exploited for protein labeling (McConnell et al., 2018). Sortase-dependent pili were thought to be exclusive to pathogens, but in the late 2000s, they were observed by transmission electron microscopy (Kankainen et al., 2009) on the non-pathogenic probiotic bacterium Lactobacillus rhamnosus GG (LGG) (Capurso, 2019), which was recently reclassified as Lacticaseibacillus rhamnosus GG (Zheng et al., 2020). When overexpressed, SpaCBA pili have also been detected on a Lactococcus lactis strain (Tarazanova et al., 2016). LGG SpaCBA pili can reach up to $1 \mu \mathrm{m}$ in length and 10 to 50 copies are found per cell (Kankainen et al., 2009). They are composed of a basal pilin (SpaB, $\sim 20 \mathrm{kDa}$ ), a major pilin (SpaA, $\sim 30 \mathrm{kDa}$ ) and a tip pilin (SpaC, $\sim 90 \mathrm{kDa})$, whose genes are clustered (Kankainen et al., 2009). The estimated molecular weight of a single pilus is 1 to $3 \mathrm{MDa}$, depending on its length. These pilins likely self-organize according to a the classical way described above to form a functional pilus (von Ossowski, 2017; Kant et al., 2020). A model of LGG pilus where SpaC is found all along the pilus was proposed following immunogold labeling and visualization of pilus structure by electron microscopy (Reunanen et al., 2012). This hypothetical model might be erroneous since polyclonal antibodies might have recognized structural motifs shared by the pilins (von Ossowski, 2017), i.e., the Ig-like fold made of seven $\beta$-sheets, of the $\mathrm{CnaB}$ domains found in the three pilins. A recent study appears to remove this ambiguity since when monoclonal antibodies were used, SpaC was only found at the tip of the pilus according to immunogold labeling in transmission electron microscopy experiments (Kant et al., 2020). Moreover, SpaCBA pili are thought to be glycosylated through $\mathrm{SpaC}$, with fucose and mannose residues (Tytgat et al., 2016). Nonetheless, SpaC harbors a structural homology with a lectin specific to mannose residues and might also contain some properties specific to fucose binding lectin (Kant et al., 2020). Therefore, the actual glycosylation of SpaC and/or its lectin capacity still need further investigation. Interestingly, chemically-induced derivatives of LGG (non-GMO) have been generated in order to improve adhesion and the associated putative probiotic role through SpaCBA pili (Rasinkangas et al., 2020).
Most studies done on LGG have aimed to decipher the interaction with molecules found in the gastro-intestinal tract (von Ossowski et al., 2010; Lebeer et al., 2012), where bacteria can exert their probiotic action. These properties include preventing pathogen adhesion, producing antimicrobial compounds (e.g., bacteriocins), fighting for nutrients, improving the epithelial barrier through mucin production, and modulating the immune response (Suez et al., 2019). Over the last decade, the use of probiotics has attracted increasing interest among consumers, whether consumed within food or as a dietary supplement. This keen interest has increased incessantly, with the ensuing pros and cons (Suez et al., 2019). One major concern when used as probiotic is to determine whether pili remain intact after industrial processes including spray-drying and the subsequent storage (Agudelo et al., 2017; Broeckx et al., 2017; Guerin et al., 2017; Gomand et al., 2019; Kiekens et al., 2019). One solution to protect bacterial pili during industrial processes consists to of encapsulating them in a dairy product-based matrix including whey proteins. This has given rise to recent studies focusing on the interaction of LGG with dairy components (Guerin et al., 2018a,b; Dos Santos Morais et al., 2020). It was demonstrated that SpaCBA pilus is capable of establishing interactions with $\beta$-lactoglobulin, while no interactions have been detected with other whey proteins (BSA and $\alpha$-lactalbumin) or with caseins (Burgain et al., 2014; Guerin et al., 2016). A divide-and-conquer approach is often employed to determine the role of each pilin subunit requiring the production of recombinant proteins (von Ossowski et al., 2010). Via this approach, structural characterizations of SpaCBA pilus subunits though X-ray crystallography (XRC) aiming to determine the structural organization are ongoing. Indeed, the XRC structure of SpaA has been made available (Chaurasia et al., 2016), such as that of SpaC (Kant et al., 2020), and efforts are currently being made to solve the structure of SpaB (Kumar Megta et al., 2019). For a better characterization of the pili function, it is essential to obtain them in a native state to take into account the potential effect of adjacent pilins, whatever the context of the gastro-intestinal tract molecules or food components.

Only few publications report the purification of native SpaCBA pili from LGG and it sometimes requires the use of specific antibodies that are non-commercially available (Reunanen et al., 2012). Others describe a centrifuge-based simple protocol to purify these pili (Tripathi et al., 2012), but exopolysaccharides (EPS) were likely co-purified during this procedure. Although the above protocol was improved by adding a size exclusion chromatography step, the required equipment is specific and costly (Tytgat et al., 2016). In the present study, an alternative protocol mainly based on multimodal chromatography (MMC, Capto Core 700) was developed. This technology combines hydrophobic interaction, ion exchange and size exclusion chromatographies, and was initially used to purify viruses (Blom et al., 2014), but it can be extended to very large molecules including pili from LGG. Furthermore, this method is straightforward and requires minimal laboratory equipment. Once purified, pili were characterized by mass spectrometry, size exclusion chromatography coupled to triple 
detection array (SEC-TDA) and successfully imaged by atomic force microscopy (AFM).

\section{MATERIALS AND METHODS}

\section{Bacterial Culture \\ Bacterial Strains and Pre-culture}

The model strain Lacticaseibacillus rhamnosus GG (ATCC53103) (LGG WT) and pili defective derivative mutant LGG $\triangle$ spaCBA:Tc ${ }^{R}$ (CMPG 5357, LGG $\triangle$ spaCBA) (Lebeer et al., 2012) were used in this study. A bacterial pre-culture was prepared by adding $500 \mu \mathrm{L}$ of bacterial glycerol stock (stored at $-80^{\circ} \mathrm{C}$ ) into $50 \mathrm{~mL}\left(\sim 1 / 100^{e}\right)$ of MRS medium (Biokar) and placed overnight at $37^{\circ} \mathrm{C}$ without agitation.

\section{Static Culture}

Lacticaseibacillus rhamnosus GG WT or LGG $\triangle$ spaCBA culture was prepared by seeding approximately $1 \mathrm{~L}$ of fresh MRS with the pre-culture $\left(\sim 1 / 50^{\circ}\right)$ and was incubated at $37^{\circ} \mathrm{C}$ without agitation until the $\mathrm{OD}_{600 \mathrm{~nm}}$ reached $\sim 1-1.2(4-5 \mathrm{~h})$. Bacteria were harvested by centrifugation $\left(3000 \times g, 10 \mathrm{~min}, 20^{\circ} \mathrm{C}\right)$. The pellets were washed with PBS pH 6.8 (Sigma) and the suspended cells were centrifuged again $\left(3000 \times \mathrm{g}, 10 \mathrm{~min}, 20^{\circ} \mathrm{C}\right)$.

\section{Bioreactor Culture}

To increase the biomass and thereby the quantity of pili, LGG WT was grown in a bioreactor under controlled $\mathrm{pH}$ (6.8, adjusted with $2 \mathrm{M} \mathrm{NaOH})$ and temperature $\left(37^{\circ} \mathrm{C}\right)$ under gentle homogenization. Five liters of MRS were seeded with $150 \mathrm{~mL}$ of pre-culture (cf. section "Bacterial Strains and Preculture"). Bacterial growth was monitored by measuring $\mathrm{OD}_{600 \mathrm{~nm}}$ every hour and by monitoring $\mathrm{NaOH}$ consumption. Cells were harvested by centrifugation $\left(3000 \times g, 10 \mathrm{~min}, 20^{\circ} \mathrm{C}\right)$ in the late exponential phase when $\mathrm{OD}_{600 \mathrm{~nm}} \sim 6-7(\sim 5 \mathrm{~h})$. They were washed with $\mathrm{PBS} \mathrm{pH} 6.8$, then centrifuged again $(3000 \times g$, $10 \mathrm{~min}, 20^{\circ} \mathrm{C}$ ).

\section{Protein Purification \\ Cell-Wall Digestion}

For clarity, Figure 1 shows a diagram summarizing the extraction and purification protocols. Washed pellets were suspended in digestion buffer (50 mM Tris pH 6.8, $150 \mathrm{mM} \mathrm{NaCl}, 2 \mathrm{mM}$ $\mathrm{MgCl}_{2}, 20 \%$ sucrose) supplemented with mutanolysin (50$100 \mathrm{U} / \mathrm{mL}$ ). The cell wall was digested overnight at $37^{\circ} \mathrm{C}$ under gentle agitation to avoid cell lysis. The measured $\mathrm{pH}$ after enzymatic digestion was 4.2 and was adjusted to 6.8 with $500 \mathrm{mM}$ Tris $\mathrm{pH}$ 6.8. The suspension was supplemented with $5 \mathrm{U} / \mathrm{mL}$ of DENARASE (C-LEcta) to digest potentially released nucleic acid material in case of eventual cell lysis, and centrifuged $(7200 \times g$, $30 \mathrm{~min}, 20^{\circ} \mathrm{C}$ ). The supernatant, containing SpaCBA pili, was carefully collected, diluted in $25 \mathrm{mM}$ Tris $\mathrm{pH}$ 6.8, and filtered through $0.45 \mu \mathrm{m}$ PES (Polyethersulphone) filter.

\section{Diafiltration}

All diafiltration steps were performed with Amicon ultra-15 concentrators (100 MWCO, Millipore) or Amicon stirred-cell
(200 mL, $100 \mathrm{MWCO}$, Millipore) under $\mathrm{N}_{2}$ pressure (2.5 bar) to handle larger volumes. Buffer exchanges differed according to the following purification step.

\section{Chromatography}

All subsequent chromatography steps were performed with an ÄKTA Start liquid chromatography system (GE Healthcare).

\section{Multimodal chromatography (MMC)}

The clarified solution was loaded at $2 \mathrm{~mL} / \mathrm{min}$ on Capto Core 700 columns (GE Healthcare) mounted in series $(5 \times 1 \mathrm{~mL}$ HiTrap column and $1 \times 4.7 \mathrm{~mL}$ HiScreen column) equilibrated with $25 \mathrm{mM}$ Tris $\mathrm{pH}$ 6.8. The flow-through was saved, then the columns were washed with the same buffer until the Abs $280 \mathrm{~nm}$ returned to the initial baseline. The columns were regenerated with $1 \mathrm{M} \mathrm{NaOH}, 30 \%$ isopropanol (cleaning in place or CIP) to desorb captured molecules.

\section{Size Exclusion Chromatography (SEC)}

Concentrated fractions $(3-5 \mathrm{~mL})$ from the flow-through of the MMC step were filtered through a $0.22 \mu \mathrm{m}$ PES filter prior to injection onto a Sephacryl HR 400 column (void volume $\sim 40 \mathrm{~mL}$, total volume $\sim 120 \mathrm{~mL}$, GE Healthcare) equilibrated with PBS pH 6.8 at $0.8 \mathrm{~mL} / \mathrm{min}$.

\section{Ion Exchange Chromatography (IEX)}

The diafiltered solution from MMC was injected onto strong anion exchange columns $(2 \times 5 \mathrm{~mL}$ HiTrap Q HP, GE Healthcare) at $5 \mathrm{~mL} / \mathrm{min}$, previously equilibrated with $25 \mathrm{mM}$ Tris pH 6.8 (buffer A). Then the columns were washed with buffer A until the Abs280nm returned to the initial baseline. Captured molecules were eluted with a linear gradient at $2 \mathrm{~mL} / \mathrm{min}$ reaching $100 \% 25 \mathrm{mM}$ Tris pH 6.8, $1 \mathrm{M} \mathrm{NaCl}$ (buffer B) in $50 \mathrm{~mL}$.

\section{SDS-PAGE Analysis and Protein Quantification}

Aliquots of each step of purification were collected and acetoneprecipitated if required. Purification steps were analyzed by SDS-PAGE (precast $4-15 \%$ or $4-20 \%$ gradient gel, Bio-Rad) in reducing condition (50 $\mathrm{mM}$ dithiothreitol, DTT) and stained with Coomassie Blue (Instant Blue, Expedeon). Protein concentration was estimated spectrometrically (NanoDrop 2000c, Thermo Scientific) considering the SpaA molar extinction coefficient $\left(39,880 \mathrm{M}^{-1} . \mathrm{cm}^{-1}\right)$ and MW $(29.2 \mathrm{kDa})$ without the signal peptide and the C-term part after the ${ }^{301} \mathrm{LPXT}^{304} \mathrm{G}$ motif $\left({ }^{34} \mathrm{DTN}\right.$...LPHT $\left.{ }^{304}\right)$. Protein purity toward other types of macromolecules was estimated considering the 260/280 ratio and visual inspection of the UV-spectrum.

\section{Mass Spectrometry}

Two different setups were employed in this study: LCMALDI and LC-ESI.

\section{LC-MALDI}

Gel bands from SDS-PAGE analysis were processed by successive washes at $20^{\circ} \mathrm{C}$ under agitation in a $50 \mu \mathrm{L}$ volume. For cysteine reduction and alkylation, bands were incubated in $100 \mathrm{mM}$ ammonium bicarbonate $(\mathrm{AB})$, then in $\mathrm{AB}$ containing $50 \mathrm{mM}$ 


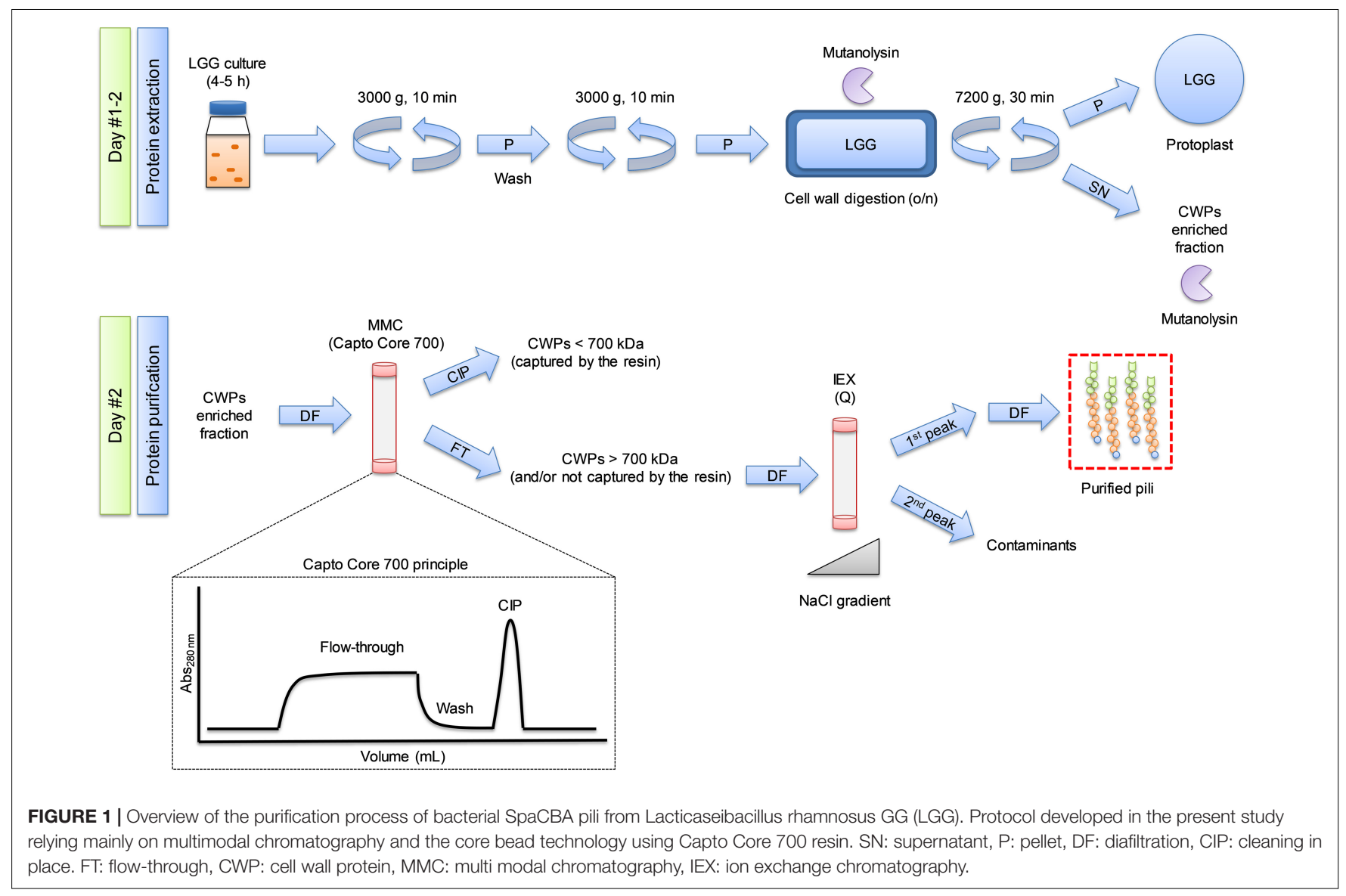

DTT for 45 min, washed once in $\mathrm{AB}$, and finally incubated in $\mathrm{AB}$ containing $50 \mathrm{mM}$ IAA (iodoacetamide) for $45 \mathrm{~min}$. Bands were washed through two cycles as follows: 15 min in $\mathrm{AB} / \mathrm{ACN}$ (acetonitrile), 1:1, $15 \mathrm{~min}$ in AB. Finally, they were dehydrated twice in $\mathrm{ACN}$ and dried in a vacuum concentrator for one hour. Bands were digested with $50 \mathrm{ng}$ trypsin (sequencing grade, Promega) overnight in $7 \mu \mathrm{L} \mathrm{AB} /$ water, 1/1. The next day, the peptides were extracted twice in $10 \mu \mathrm{L}$ ACN, 80\%, TFA $1 \%$ for $7 \mathrm{~min}$ under sonication. This procedure was done twice. Peptide extracts were pooled and dried in a vacuum concentrator, resuspended in $10 \mu \mathrm{l} 2 \% \mathrm{ACN}, 0.1 \%$ TFA and processed for fractionation by nano HPLC.

Nano HPLC was carried out with a UltiMate 3000 system (Thermo Scientific) equipped with a $20 \mu \mathrm{L}$ sample loop, a pepMap $100 \mathrm{C} 18$ desalting precolumn (Dionex) and a $15 \mathrm{~cm}$ pepMap RSLC C18 fractionation column (Dionex). Samples ( $5 \mu \mathrm{L}$ ) were injected using the $\mu \mathrm{L}$ pickup mode and eluted by a 2 to $45 \%$ ACN gradient over $30 \mathrm{~min}$ at $300 \mathrm{nl} / \mathrm{min}$. Fractions (170, $9 \mathrm{~s}$ each) were collected on a ProteineerFcII (Bruker) over $25.5 \mathrm{~min}$ and eluates were directly mixed on MTP-1536 TF target (Bruker) spots to $\alpha$-cyano-4-hydroxycinnamic acid (Bruker). LCMALDI runs were processed using dedicated automatic methods piloted by WARP-LC software on an Autoflex speed MALDITOF/TOF mass spectrometer (Bruker) in the 800-3,500 mass range, using next-neighbor external calibration for all MALDI spots, using 2000 random laser shots per spot at a $2000 \mathrm{~Hz}$ frequency. Masses detected with $\mathrm{S} / \mathrm{N}$ above 20 were selected for TOF/TOF fragmentation in LIFT mode.

Peptide assignments were performed from TOF/TOF spectra by Mascot Server interrogation (v2.4.1, Matrix Science) piloted and compiled by Proteinscape. The database search parameters were as follows: mass tolerance for precursors $=50 \mathrm{ppm}$; mass tolerance for fragments $=0.8 \mathrm{Da}$; enzyme $=$ trypsin with one missed cleavage allowed; protein modifications = carbamidomethylation of cysteines (variable) and oxidation of methionines (variable). The specific database was [Uniprot proteome UP000000955, 2019/06/28, 2877 sequences, (Kankainen et al., 2009)]. Proteins were considered as identified when at least two peptides passed the Mascot score with $p<0.05$ threshold.

\section{LC-ESI}

Gel pieces from SDS-PAGE were excised and cut into small cubes and processed with the in-gel digestion kit (Thermo Scientific). Briefly, the gels pieces were destained with acetonitrile 30\%, reduced with TCEP [tris(2-carboxyethyl)phosphine], alkylated with IAA and digested with trypsin overnight at $37^{\circ} \mathrm{C}$. The peptides were extracted according to a previously published method (Lavigne et al., 2012).

Peptides were analyzed on a Vanquish quaternary UHPLC system (Thermo Scientific) in-line with a photodiode array detector (PDA) and an Orbitrap ID-X Tribrid mass spectrometer 
(Thermo Scientific) equipped with an atmospheric pressure ionization interface operating in electrospray mode (ESI). Sixteen microliters of peptides were separated on an Acclaim 120 C18 column $(100 \mathrm{~mm} \times 2.1 \mathrm{~mm}-2.2 \mu \mathrm{m}$, Thermo Scientific) maintained at $30^{\circ} \mathrm{C}$. The flow rate was set at $200 \mu \mathrm{l} / \mathrm{min}$ and mobile phases consisted in water modified with formic acid $(0.1 \%)$ for $\mathrm{A}$ and acetonitrile modified with formic acid $(0.1 \%)$ for B. Peptides were eluted using a gradient step of 5 to $95 \%$ B for $40 \mathrm{~min}$, then an isocratic step was applied at $95 \%$ B for $10 \mathrm{~min}$ to wash the column, before returning to the initial composition of $5 \% \mathrm{~B}$ for $5 \mathrm{~min}$ to realize the equilibrium. Mass analysis was carried out in ESI positive ion mode $($ ESI +$)$ and mass spectrometry conditions were as follows: spray voltage was set at $3.5 \mathrm{kV}$; source gases were set (in arbitrary units/min) for sheath gas, auxiliary gas and sweep gas at 35,7 , and 0 , respectively; vaporizer temperature and ion transfer tube temperature were both set at $300^{\circ} \mathrm{C}$. Survey scans of peptide precursors from 150 to $2,000 \mathrm{~m} / \mathrm{z}$ were performed at $60 \mathrm{~K}$ resolution (full width of the peak at its half maximum, fwhm, at $200 \mathrm{~m} / \mathrm{z}$ ) with MS parameters as follows: RF-lens, 35\%; maximum injection time, $50 \mathrm{~ms}$; data type, profile; internal mass calibration EASY-IC TM activated; custom AGC target; normalized AGC target: 25\%. A top speed (0.6 s) data-dependent MS2 was performed by isolation at 1.5 Th with the quadrupole, HCD fragmentation with a stepped collision energy $(25,35$, and 50) and MS analysis in the Orbitrap at $15 \mathrm{~K}$ resolution (high resolution MS/MS analysis). Only the precursors with intensities above the threshold of 2.104 were sampled for MS2. The dynamic exclusion duration was set to $2.5 \mathrm{~s}$ with a $10 \mathrm{ppm}$ tolerance around the selected precursor (isotopes excluded). Other MS2 parameters were as follows: data type, profile; custom AGC target; normalized AGC target: $20 \%$. Mass spectrometer calibration was performed using the Pierce FlexMix calibration solution (Thermo Scientific). MS data acquisition was carried out utilizing the Xcalibur v. 3.0 software (Thermo Scientific).

LC-MSMS data were processed and analyzed with Mascot Distiller (v2.7, Matrix Science) and Mascot Server (v2.7, Matrix Science). The database search parameters were as follows: mass tolerance for precursors $=10 \mathrm{ppm}$; mass tolerance for fragments $=20 \mathrm{ppm}$; enzyme $=$ trypsin with two missed cleavages allowed; protein modifications $=$ carbamidomethylation of cysteines (fixed) and oxidation of methionines (variable). The databases were [\#1: Contaminants 2016/01/29, 247 sequences, \#2: SwissProt 2019/11, 561568 sequences, and \#3: Uniprot_LGG_UP000000955, 2020/02/25, 2877 sequences, (Kankainen et al., 2009)]. Proteins were considered as identified when at least two peptides passed the Mascot score with $p<0.01$ threshold scores.

\section{Dynamic Light Scattering (DLS)}

The polydispersity and the hydrodynamic radius $\left(R_{h}\right)$ of the pili were estimated by DLS using a Zetasizer instrument (Nano ZS, Malvern Panalytical). The sample was filtered through a $0.22 \mu \mathrm{m}$ PES-filter just before analysis. Measurements were performed at $20^{\circ} \mathrm{C}$ in PBS buffer ( $\mathrm{pH} 7.4$ ) using a quartz cell. Data were processed with the Zetasizer software (v7.13, Malvern Panalytical) with default parameters. The size distribution by intensity was obtained from 6 successive experiments to ensure sample stability.

\section{Size-Exclusion Chromatography Coupled to Triple Detection Array (SEC-TDA)}

Size exclusion chromatography experiments were performed with a HPLC pump (LC10AD, Shimadzu) coupled to an autosampler (Viscotek VE 2001, Malvern Panalytical) and a multi-detector system recording light scattering (RALS/LALS) intrinsic viscosity and refractive index signals (Viscotek TDA305, Malvern Panalytical). The HPLC SEC column (BioSec5 $500 \AA$, $5 \mu \mathrm{m}, 7.8 \mathrm{~mm}$ ID $\times 300 \mathrm{~mm}$, void volume $\sim 6 \mathrm{~mL}$, total volume $\sim 12.5 \mathrm{~mL}$, Agilent) was equipped with a post-column nylon filter $(0.22 \mu \mathrm{m})$. The column was equilibrated with PBS $(\mathrm{pH}$ 6.8) supplemented with sodium azide $0.02 \%$. The flow rate and the temperature were $0.35 \mathrm{~mL} / \mathrm{min}$ and $30^{\circ} \mathrm{C}$ respectively. Data were processed with the OmniSEC software (v5.12, Malvern Panalytical). The calibration procedure was done with BSA (Sigma) and cross-validation was performed with $\beta$-lactoglobulin (Sigma). The refractometer was used as the concentration detector and the refractive index increment value $(d n / d c)$ used to determine the molecular weight was $0.185 \mathrm{~mL} / \mathrm{g}$. Samples were diafiltered in the aforementioned buffer and filtered through a $0.22 \mu \mathrm{m}$ PES-filter just before injection.

\section{Atomic Force Microscopy (AFM) Imaging}

Two hundred microliters of LGG WT cell suspension cultivated as in section "Bacterial Strains and Pre-Culture" in MRS or two hundred microliters of purified pili (diluted at 50 and $2.5 \mathrm{mg} / \mathrm{L}$ ) in $25 \mathrm{mM}$ Tris $\mathrm{pH}$ 6.8, $150 \mathrm{mM} \mathrm{NaCl}$ were deposited on freshly cleaved mica substrates and allowed to settle for $2 \mathrm{~h}$. Then the surfaces were gently rinsed by immersion in 3 baths of ultrapure water and dried overnight at $30^{\circ} \mathrm{C}$ before imaging. Images were obtained in peak force tapping mode with a Bioscope Resolve AFM (Bruker corporation, Santa Barbara, CA, United States), using SNL cantilevers (Bruker corporation, nominal spring constant of $\sim 0.24 \mathrm{~N} . \mathrm{m}^{-1}$ ) and with a maximum applied force of $5 \mathrm{nN}$.

\section{RESULTS AND DISCUSSION}

\section{Purification Protocols Available in the Literature}

The literature was investigated to determine available protocols to purify pili from LGG. First, (Tripathi et al., 2012) developed a simple centrifuged-based protocol. The bacterial culture was subjected to two successive centrifugation steps, one at $8000 \times g$ to remove pili from bacteria due to shear stress and a second one at $20000 \mathrm{~g}$ to pellet them. The authors characterized the pili by AFM and admitted that exopolysaccharides (EPS) could have co-precipitate during the $20000 \times g$ centrifuge step, meaning that EPS contaminants could interfere with other techniques. Moreover, other cell wall proteins (CWPs) could have been released during the centrifugation steps and probably altered the 
pili purity even if they were not visible by AFM, due to their relative small sizes.

In the study by Tytgat et al. (2016) LGG was grown in an industrial whey permeate, as previously done (Laakso et al., 2011), and cells were concentrated by microfiltration. Cells were then centrifuged at $20000 \times g$, and the authors stated that the pili were found in the supernatant instead of the pellet, contrary to Tripathi et al. (2012). Then they added a second step of purification consisting of a size exclusion chromatography step. Pili were presumably found in the void volume of the column. In this case, EPS might have co-eluted with the pili during this step and SEC requires specific equipment.

Instead of simply centrifuging bacterial cells, (Reunanen et al., 2012) digested the cell-wall with mutanolysin and lysozyme in order to release CWPs including pili. Then CWPs were injected onto a column functionalized with polyclonal antibodies antiSpaCBA, allowing them to remove contaminants. It is worth noting that to destabilize the specific antibody-antigen bound, it is necessary to elute the bound proteins at low $\mathrm{pH}(\sim 3)$. This could damage the native state of the pili even if $\mathrm{pH}$ was neutralized directly in collection tube. Although efficient, this protocol requires the use of specific antibodies that are not commercially available. Moreover, the intermediates of polymerization were still present in the purified fractions, as shown by western blots.

\section{Extraction of SpaCBA Pili via Enzymatic Digestion}

In order to purify the pili, the simplest protocol available (i.e., the centrifuge-based protocol) developed by Tripathi et al. (2012) and improved by Tytgat et al. (2016) was tested. Unfortunately, for us it was not possible to extract any detectable CWPs in the supernatant or in the pellet of each centrifuge steps based on Coomassie blue staining after SDS-PAGE analysis. The same result was obtained after resuspending the cells vigorously with a vortex during $1 \mathrm{~min}$ before the $8000 \times g$ centrifuge step (Supplementary Figure 1A).

As a second option, it was decided to employ a double enzymatic digestion of the CW with mutanolysin and lysozyme, as done by Reunanen et al. (2012) (Supplementary Figure 1B). Mutanolysin being quite expensive, the enzyme concentration was lowered to $50-100 \mathrm{U} / \mathrm{mL}$ and the digestion time increased from $2 \mathrm{~h}$ to overnight $(\sim 12 \mathrm{~h})$ under gentle agitation to avoid protoplast lysis. Later, only mutanolysin was employed as it was not necessary to use lysozyme to obtain a satisfactory CW digestion (data not shown). As expected, the enzymatic digestion of LGG CW released CWPs, as previously done to purify pili from Streptococcus pneumoniae (Hilleringmann et al., 2008).

For the development of the protocol of purification, LGG WT was cultivated in MRS medium in static condition under uncontrolled $\mathrm{pH}$, and LGG $\triangle s p a C B A$ was used as a negative control. The difference in migration in SDS-PAGE, only visible at the top of the gel, clearly indicated the absence of pili on LGG $\triangle$ spaCBA (Figure 2A). This approach would allow for a rapid identification of pili at the protein level for other lactic acid bacteria. Currently, the presence/absence of pili is limited to genome mining and analysis, without any guarantee of gene expression and protein production. To increase the biomass and the quantity of pili, LGG WT was grown in a bioreactor under controlled $\mathrm{pH}$ (6.8) and gentle homogenization. For both static and bioreactor cultures, cells were harvested by centrifugation in the late-exponential growth phase where pili are still expressed (Laakso et al., 2011).

\section{Purification of SpaCBA Pili via Chromatography}

Having a CWP-enriched fraction, the chosen approach to separate pili from other protein contaminants was ratiocinated based on their size. Considering the length of SpaA $(\sim 9.3 \mathrm{~nm})$ (Chaurasia et al., 2016), and taking into account the fact that the size of pili ranges from $\sim 300 \mathrm{~nm}$ to up to $1 \mu \mathrm{m}$, it was roughly estimated that a pilus is composed of $\sim 33-100$ SpaA subunits. The MW of SpaA being $\sim 30 \mathrm{kDa}$, it can be reasonably assumed that a single pilus has a MW of 1-3 MDa. With this information in mind, the use of multimodal chromatography (MMC, Capto Core 700) to purify the pili was chosen. This core bead technology was initially developed for virus purification (Blom et al., 2014), but should be adapted for the purification of high molecular weight (HMW) proteins including bacterial pili (Figure 1). This technique is a flow-through-based method. Molecules having a $\mathrm{MW}>$ to $700 \mathrm{kDa}$ cannot enter the beads while molecules having MW < to $700 \mathrm{kDa}$ should enter the beads and be captured via electrostatic and/or hydrophobic interactions via the octyl amine ligand that is positively charged at neutral $\mathrm{pH}$. Positively charged molecules that are not retained by the hydrophobic part of the ligand will also go through the beads. Up to 10 column volume (CV) can be loaded onto the column, much more than the $0.5-4 \%$ $\mathrm{CV}$ of sample volume that is recommended to be loaded on SEC columns. This unequivocally increases the amount of samples as well as the rapidity of the purification. Notably, the technology is available for $400 \mathrm{kDa}$ molecules (Capto Core 400).

The supernatant of digestion (SOD) was injected on Capto Core 700 columns and the flow-through was saved. The column was washed with PBS and regenerated with $\mathrm{NaOH}$ /isopropanol mixture to desorb simultaneously molecules bound via hydrophobic and/or electrostatic interactions (Figure 2B). Following SDS-PAGE analysis (Figure 2D), one smeared band in the HMW part on the gel $(>260 \mathrm{kDa})$ was observed and other bands from 30 to $60 \mathrm{kDa}$ were noticed in the flow-through fraction. In the cleaning-in-place fraction, a smear was noted down to $10 \mathrm{kDa}$, meaning that plenty of CWPs were successfully bound to Capto Core 700 resin. Importantly, it was noted that running SDS-PAGE at low current $(\sim 100 \mathrm{~V})$ improved the resolution at HMW and gave a smeared band as expected, corresponding to pili with different but close sizes. Interestingly, the major pilin SpaA harbors two isopeptide bonds between K47 and N172 and K184 and D295, one in each CnaB domain (Chaurasia et al., 2016). If those bonds are maintained during SDS-PAGE analysis, it could drastically decrease the apparent MW of the pili since the proteins might not be fully unfolded by SDS. Indeed, $\mathrm{CnaB}$ domains are predicted to be inextensible, contrary to CnaA domains, due to the difference in position of the 


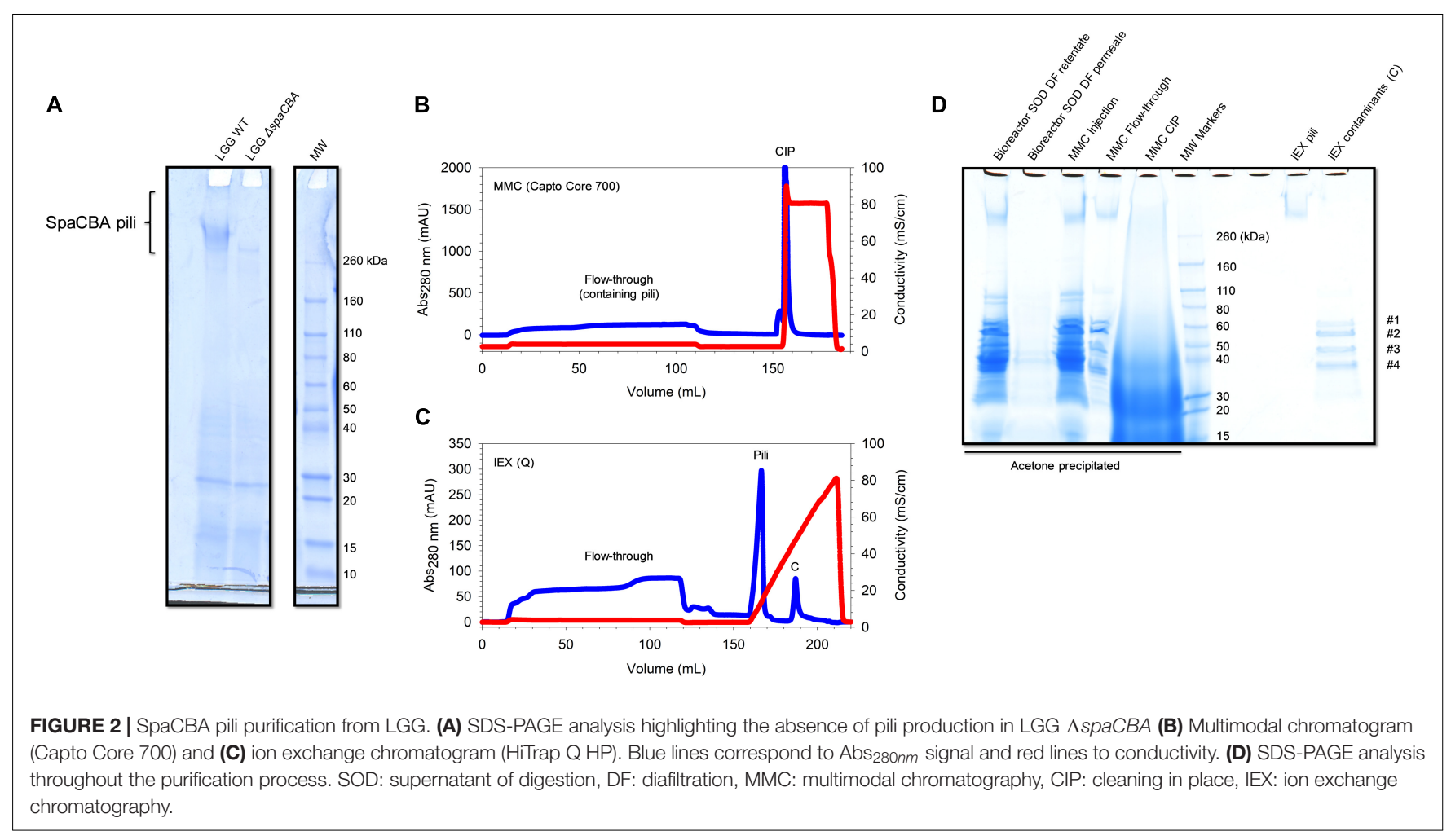

isopeptide bonds (Echelman et al., 2016). From one purification batch, a band at HMW was excised, trypsin digested and analyzed by mass spectrometry (LC-MALDI) (Supplementary Table 1). Six peptides matching with SpaC sequence (UniParc: UPI0001B5E4D6) were identified, confirming the presence of the pilin and therefore of pili. These peptides are found either in the CnaA or the vWFA domains (PDB: 6M48). Moreover, two peptides belonging to a protein of unknown function (matrix-binding protein, UniParc: UPI0001B5E7B8) were also identified. Surprisingly, only one peptide from SpaA (UniParc: UPI0001B5E4D4) was found in a $\beta$-sheet from one CnaB domain (PBD: 5F44). However, it did not pass the conditions confirming the presence of the protein, i.e., identifying at least two peptides having a score higher than the threshold $(p<0.05)$. This result was quite surprising, since SpaA is the major pilin and should be the most represented in the pilus. One explanation might be the resistance to trypsin digestion since the presence of the two isopeptide bonds could drastically affect digestion efficiency if they maintain a large portion of intact secondary structures, and so a resistance to SDS unfolding similarly to mechanical unfolding (Baker et al., 2015; Chaurasia et al., 2016). One peptide belonging to a murein DD-endopeptidase (UniParc: UPI0001B5E883) was also identified. Not surprisingly, no peptides from $\mathrm{SpaB}$ were detected since it is the least represented pilin within the pilus.

From another purification batch after MMC, it was observed that most of the CWPs were again efficiently captured by Capto Core 700 resin and only five bands were detected by SDS-PAGE: the band found at HMW, likely the pili, and four bands at $35,50,60$, and $65 \mathrm{kDa}$ (Figure 2D). In order to eliminate the low molecular weight contaminants, concentrated samples were injected from the MMC step onto a SEC column (Sephacryl HR 400, separation range for globular proteins: $20-8000 \mathrm{kDa}$ ). Only one peak was observed as having an elution volume (Ve) of $\sim 40 \mathrm{~mL}$. After SDS-PAGE analysis, contaminants were still present (data not shown). Thus, it was suggested that these contaminants might co-elute with the pili, meaning that they interact with each other, or that they just have the same elution volume. To answer the question, the same protocol was used to purify CWPs from LGG $\triangle$ spaCBA mutant, which is devoid of pili. After CW digestion, MMC and SEC, bands at $\sim 35,50$, 60 , and $65 \mathrm{kDa}$ were still observed but no longer the band at HMW, confirming that this band corresponds effectively to the pili (data not shown). Moreover, these contaminants had the same elution volume on SEC, meaning that they do not bind pili but are certainly a complex or aggregate of HMW. To obtain more information about these proteins, they were analyzed by mass spectrometry (LC-ESI). All of them were successfully identified as ABC transporter substrate binding-related proteins (Supplementary Table 2).

To get rid of these protein contaminants and to concentrate SpaCBA pili, IEX chromatography was used. SpaA has a predicted pI of 4.7 in its mature form, i.e., without the signal peptide and without the C-term part after the LPXTG motif. Therefore, pili should bind to an anion-exchange column, while the contaminants have a $\mathrm{pI}>9$ and should not bind to the resin. Before injecting the flow-through from MMC, the ionic strength of the sample was lowered by diafiltration in Tris $25 \mathrm{mM} \mathrm{pH} 6.8$ buffer to ensure a proper binding of the pili. The diafiltered sample was injected on strong anion 
exchange chromatography bearing a quaternary ammonium group. Once the sample was completely loaded and the columns washed, proteins were eluted with a linear gradient to $1 \mathrm{M}$ $\mathrm{NaCl}$ in $5 \mathrm{CV}$. Two properly separated peaks (Figure 2C) were observed, one eluting at $\sim 15 \mathrm{mS} / \mathrm{cm}$ and the other at $50 \mathrm{mS} / \mathrm{cm}$ (top of the peaks). The first peak contained the pili and the second one the contaminants, as shown in SDS-PAGE analysis (Figure 2D). The contaminants were not expected to remain bound to the resin since the theoretical pI is $>9$ and those proteins should have been positively charged. Positive charges may be hidden while negative charges are accessible in the conformation state adopted by these $\mathrm{ABC}$ transporter-related complex/aggregates. Although no other protein other than pili were detected on SDS-PAGE stained with Coomassie blue, UV-vis quantification showed an unusual $260 \mathrm{~nm} / 280 \mathrm{~nm}$ ratio $>1$, showing that non-protein biomolecules were present with the purified pili. A simple last step of diafiltration was added to obtain highly purified pili to get rid of these contaminants.

Although the IEX steps were performed with an automatic liquid chromatography device, peaks were well separated, and operating with a syringe in step elution mode, instead of gradient, should not interfere with pili purity. Interestingly, keeping the ionic strength high after the MMC step ( $\sim 15 \mathrm{mS} / \mathrm{cm})$ allowed the pili to flow through the column, but the contaminants were still captured. In this case, the pili were highly diluted but could easily be concentrated afterward (data not shown). Other conditions were tested during the development of this protocol including running MMC in PBS pH 7.4 with or without $300 \mathrm{mM} \mathrm{NaCl}$, but this did not change the results. Similarly, running $\mathrm{MMC}$ in Tris buffer $25 \mathrm{mM}$ with $150 \mathrm{mM} \mathrm{NaCl}$ did not capture more protein contaminants and a second step of IEX was mandatory to obtain purified pili. To sum up, a simple protocol based mainly on MMC that allows for the purification of SpaCBA pili from LGG was developed. In 2-3 working days, and starting from $1 \mathrm{~L}$ culture at $\mathrm{OD}_{600 \mathrm{~nm}} \approx 1, \sim 50 \mu \mathrm{g}$ of pili ( $\sim 2.10^{13}$ molecules, $500 \mathrm{~nm}$ long, $\left.1.6 \mathrm{MDa}\right)$ can be purified. $\mathrm{OD}_{600 \mathrm{~nm}} \approx 1$ corresponds to a bacterial concentration of $\sim 1.25 .10^{8} \mathrm{CFU} / \mathrm{mL}$ [considering 5 LGG per bacterial chain (Gomand et al., 2020)]. This suggests that $\sim 30$ pili are purified per bacterium.
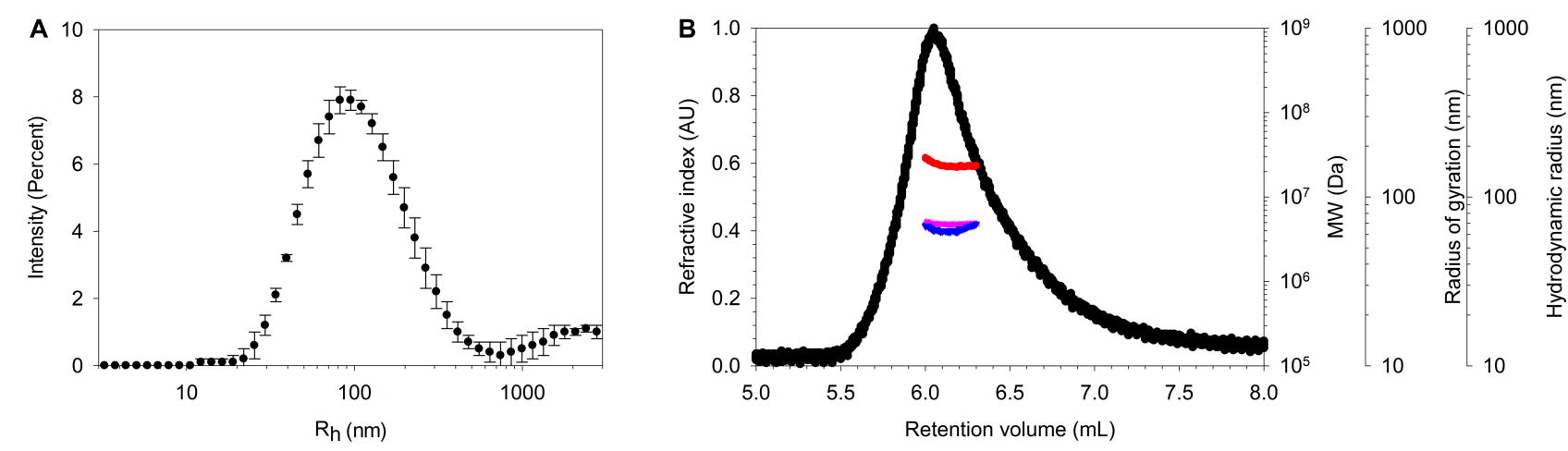

FIGURE 3 | Characterization of the purified pili. (A) DLS analysis showing a large distribution of $\mathrm{R}_{h}$ centered on $\sim 85 \mathrm{~nm}$. (B) SEC-TDA analysis of SpaCBA pilus giving a MW (red) at the top of the peak of $\sim 25 \mathrm{MDa}$ and $\mathrm{R}_{g}$ (pink) and $\mathrm{R}_{h}$ (blue) values of 70 and $65 \mathrm{~nm}$. Refractive index signal is represented in black. The void volume and the total volume of the SEC column are $\sim 6$ and $12.5 \mathrm{~mL}$ respectively.
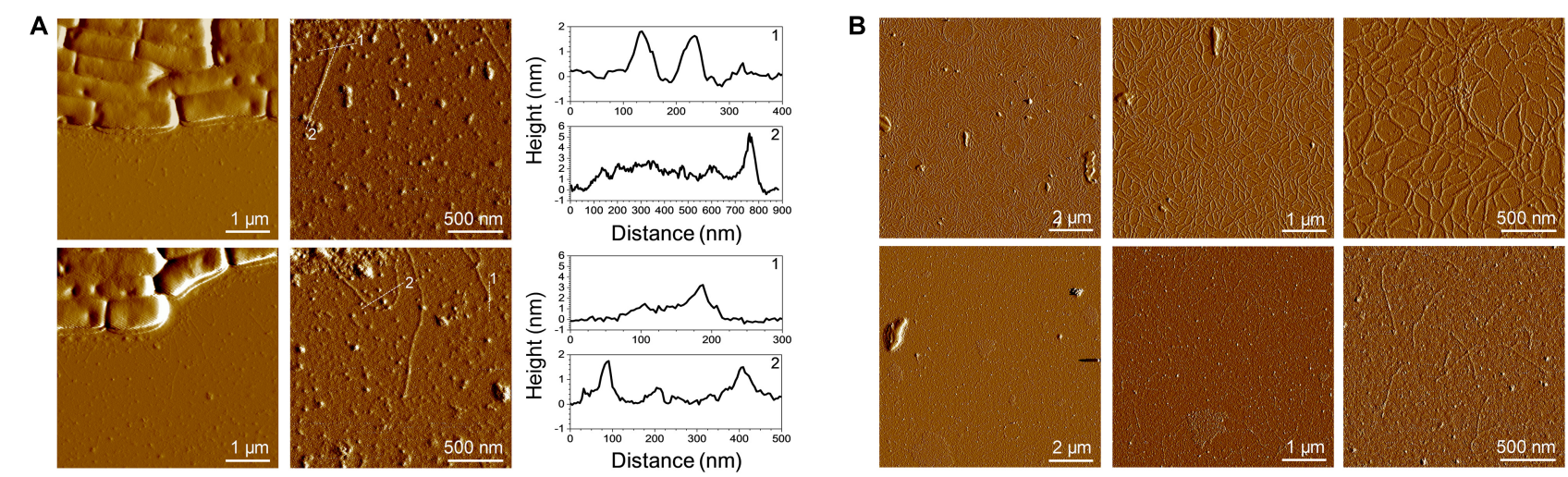

FIGURE 4 | AFM imaging of SpaCBA pili. (A) Low and high resolution images of LGG cells presenting pili. The vertical cross sections were taken along the dashed lines on the corresponding height images. (B) Purified pili imaged at two concentrations (top: $50 \mathrm{mg} / \mathrm{L}$, bottom: $2.5 \mathrm{mg} / \mathrm{L}$ ). 


\section{Characterization of the Purified SpaCBA Pili}

SpaCBA pili, purified thanks to this method, were characterized by DLS (Figure 3A). The mean $R_{h}$ is centered at $82 \mathrm{~nm}$ and the distribution is broad since a polydispersity index (PDI) $>0.7$ was obtained, as expected. Indeed, pili could harbor different hydrodynamic volumes according to the number of SpaA found in one pilus and/or they may adopt a conformation that is more or less straight or bent in solution. The purified pili were then analyzed by HPLC, more precisely by SEC coupled to multidetectors (TDA) to determine their MW, radius of gyration $\left(R_{g}\right)$ and hydrodynamic radius $\left(R_{h}\right)$ (Figure 3B). Buffer exchange was performed by diafiltration with the same buffer as the eluent to avoid buffer mismatches that could interfere with the refractometer signal at the end of the column. The first observation was that the pili eluted close to the void volume $(\sim 6 \mathrm{~mL})$ of the column designed to separate globular proteins up to $5 \mathrm{MDa}$, confirming their high $\mathrm{MW}$. The $\mathrm{MW}$ value at the top of the peak was $\sim 25 \mathrm{MDa}$ while $R_{g}$ and $R_{h}$ values were $\sim 70$ and $\sim 65 \mathrm{~nm}$ respectively. Henceforth, the shape factor $\rho$ $\left(R_{g} / R_{h}\right)$ was approximately 1.1 , a value in line with the elongated form of the pili. From these data, it can be concluded that pili are not monomeric and might form aggregates as previously observed by Tripathi et al. (2012), who observed by AFM the formation of bundles. Differences in $R_{h}$ could be due to a small proportion or larger pili present in DLS analysis, but not in SECTDA analysis, since the latter is equipped with a post-column nylon filter. Moreover, SEC-TDA data are from the top of the peak only and did not include the analysis of the whole peak.

\section{AFM Imaging of Dry Pili}

Atomic force microscopy was employed for further characterization and imaging. Imaging pili is quite challenging and only a few publications have reported AFM imaging of these filamentous proteins. Indeed, LGG pili were successfully imaged directly on bacteria or after purification (Tripathi et al., 2012). Recently, the resolution serving to distinguish SpaA all along the pilus and $\mathrm{SpaC}$ at the tip was even reached (Kant et al., 2020). In the present work, pili were imaged on bacteria as well (Figure 4A). As expected, they appear to be protruding, long and thin filaments having a length around $500 \mathrm{~nm}$. The height measured on a pilus on a cross-section from the base to the tip corresponds to a thickness of $2 \mathrm{~nm}$ all along the pilus and a value of around $4-5 \mathrm{~nm}$ at the top of the pilus. These values are in line with those of the aforementioned recently published work (Kant et al., 2020). Purified pili were also imaged by AFM (Figure 4B). Pili were analyzed either at 50 or at $2.5 \mathrm{mg} / \mathrm{L}$. At the higher concentration, the pili formed a network bound and no bundles were observed as previously reported (Tripathi et al., 2012). Such bundles could have been formed due to the copurification of EPS following the centrifugation-based protocol (Tripathi et al., 2012; Supplementary Figure 1A). Nonetheless, a thickness of $\sim 2 \mathrm{~nm}$ was measured. This value is in line with the XRC 3D structure of SpaA, the major pilin (Chaurasia et al., 2016). At this concentration and taking into account drying, this network formation might occur via SpaC since
SpaC-SpaC interactions were highlighted by single molecule force spectroscopy with recombinant SpaC (Tripathi et al., 2013). At the lower concentration, isolated purified pili are easily detectable and, similarly to cell-attached pili, the average length was $500 \mathrm{~nm}$ and the thickness $2 \mathrm{~nm}$ (Figure 4B). No measures at the top of the purified pilus were made since the $\mathrm{N}$-term C-term orientation might be tricky and could have led to mistakes.

\section{CONCLUSION}

In the present study, we have developed a more versatile and easy to implement protocol than those available in the literature to purify SpaCBA pili from Lacticaseibacillus rhamnosus GG, one of the most widely studied probiotic bacteria, whose probiotic properties might partially occur through these pili. This novel protocol is mainly based on multimodal chromatography (core bead technology using Capto Core 700 resin), requires minimal laboratory equipment, is not expensive and can be used to obtain SpaCBA pili with a high degree of purity. Although done with a basic chromatographic system, purification might be performed with a peristaltic pump or even a simple syringe and would not require complex lab equipment nor the use of specific antibodies that are not commercially available. A custom antibody production might be expensive and only dedicated to the targeted pilins. This work could allow for the expansion of studies dedicated to SpaCBA pili at the molecular level, and the characterization of interactions with their protein partners would also be facilitated. Finally, we believe that this new purification method might be a solid basis for further purification of other sortase-dependant pili.

\section{DATA AVAILABILITY STATEMENT}

The mass spectrometry proteomics data have been deposited to the ProteomeXchange Consortium via the PRIDE (PerezRiverol et al., 2019) partner repository with the dataset identifier PXD020875.

\section{AUTHOR CONTRIBUTIONS}

RDSM, JB, FB, and CG designed the study and wrote the manuscript. RDSM, BS, and SB prepared the sample. RDSM, SE$\mathrm{K}-\mathrm{C}, \mathrm{BS}$, and CP acquired the data. RDSM and SE-K-C analyzed or interpreted the data. RDSM drafted the manuscript. All authors reviewed the manuscript and approved the final version.

\section{FUNDING}

We are grateful for the financial support of the French ANR - Agence Nationale de la Recherche (ANR-18-CE210003). Furthermore, we acknowledge financial support from the "Impact Biomolecules" project of the "Lorraine Université 
d'Excellence" (Investissements d'avenir - ANR 15-004). LGG WT and $\triangle s p a C B A$ mutant were kind gifts from Dr. Sarah Leeber.

\section{ACKNOWLEDGMENTS}

We are grateful to Dr. Emmanuel Rondags for the culture in the bioreactor. We would like to thank Dr. Jean-Baptiste Vincourt from the Proteomics Core Facility of UMS2008/US40 IBSLor

\section{REFERENCES}

Agudelo, J., Cano, A., González-Martínez, C., and Chiralt, A. (2017). Disaccharide incorporation to improve survival during storage of spray dried Lactobacillus rhamnosus in whey protein-maltodextrin carriers. J. Funct. Foods 37, 416-423. doi: 10.1016/j.jff.2017.08.014

Baker, E. N., Squire, C. J., and Young, P. G. (2015). Self-generated covalent crosslinks in the cell-surface adhesins of Gram-positive bacteria. Biochem. Soc. Transact. 43, 787-794. doi: 10.1042/BST20150066

Berne, C., Ellison, C. K., Ducret, A., and Brun, Y. V. (2018). Bacterial adhesion at the single-cell level. Nat. Rev. Microbiol. 16, 616-627. doi: 10.1038/s41579-018005755

Blom, H., Åkerblom, A., Kon, T., Shaker, S., van der Pol, L., and Lundgren, M. (2014). Efficient chromatographic reduction of ovalbumin for egg-based influenza virus purification. Vaccine 32, 3721-3724. doi: 10.1016/j.vaccine.2014. 04.033

Broeckx, G., Vandenheuvel, D., Henkens, T., Kiekens, S., van den Broek, M. F. L., Lebeer, S., et al. (2017). Enhancing the viability of Lactobacillus rhamnosus GG after spray drying and during storage. Int. J. Pharmac. 534, 35-41. doi: 10.1016/j.ijpharm.2017.09.075

Burgain, J., Scher, J., Lebeer, S., Vanderleyden, J., Cailliez-Grimal, C., Corgneau, M., et al. (2014). Significance of bacterial surface molecules interactions with milk proteins to enhance microencapsulation of Lactobacillus rhamnosus GG. Food Hydrocol. 41, 60-70. doi: 10.1016/j.foodhyd.2014.03.029

Capurso, L. (2019). Thirty Years of Lactobacillus rhamnosus GG: A Review. J. Clin. Gastroenterol. 53:S1. doi: 10.1097/MCG.0000000000001170

Chang, C., Amer, B. R., Osipiuk, J., McConnell, S. A., Huang, I.-H., Hsieh, V., et al. (2018). In vitro reconstitution of sortase-catalyzed pilus polymerization reveals structural elements involved in pilin cross-linking. PNAS 115, E5477-E5486. doi: 10.1073/pnas.1800954115

Chaurasia, P., Pratap, S., von Ossowski, I., Palva, A., and Krishnan, V. (2016). New insights about pilus formation in gut-adapted Lactobacillus rhamnosus GG from the crystal structure of the SpaA backbone-pilin subunit. Sci. Rep. 6:28664. doi: 10.1038/srep28664

Dos Santos Morais, R., Gaiani, C., Borges, F., and Burgain, J. (2020). Interactions Microbe-Matrix in Dairy Products. Reference Module in Food Science. Netherland: Elsevier.

Echelman, D. J., Alegre-Cebollada, J., Badilla, C. L., Chang, C., Ton-That, H., and Fernández, J. M. (2016). CnaA domains in bacterial pili are efficient dissipaters of large mechanical shocks. PNAS 113, 2490-2495. doi: 10.1073/ pnas. 1522946113

Gomand, F., Borges, F., Burgain, J., Guerin, J., Revol-Junelles, A. M., and Gaiani, C. (2019). Food Matrix Design for Effective Lactic Acid Bacteria Delivery. Annu. Rev. Food Sci. Technol. 10, 285-310. doi: 10.1146/annurev-food-032818121140

Gomand, F., Mitchell, W. H., Burgain, J., Petit, J., Borges, F., Spagnolie, S. E., et al. (2020). Shaving and breaking bacterial chains with a viscous flow. Soft. Matter 16, 9273-9291. doi: 10.1039/D0SM00292E

Guerin, J., Bacharouche, J., Burgain, J., Lebeer, S., Francius, G., Borges, F., et al. (2016). Pili of Lactobacillus rhamnosus GG mediate interaction with $\beta$-lactoglobulin. Food Hydrocolloids 58, 35-41. doi: 10.1016/j.foodhyd.2016.02.016

Guerin, J., Burgain, J., Francius, G., El-Kirat-Chatel, S., Beaussart, A., Scher, J., et al. (2018a). Adhesion of Lactobacillus rhamnosus GG surface biomolecules to milk proteins. Food Hydrocolloids 82, 296-303. doi: 10.1016/j.foodhyd.2018.04.016
(Université de Lorraine, CNRS, INSERM), Nancy, France for LC-MALDI data analysis.

\section{SUPPLEMENTARY MATERIAL}

The Supplementary Material for this article can be found online at: https://www.frontiersin.org/articles/10.3389/fmicb. 2020.609880/full\#supplementary-material

Guerin, J., Petit, J., Burgain, J., Borges, F., Bhandari, B., Perroud, C., et al. (2017). Lactobacillus rhamnosus GG encapsulation by spray-drying: Milk proteins clotting control to produce innovative matrices. J. Food Engin. 193, 10-19. doi: 10.1016/j.jfoodeng.2016.08.008

Guerin, J., Soligot, C., Burgain, J., Huguet, M., Francius, G., El-Kirat-Chatel, S., et al. (2018b). Adhesive interactions between milk fat globule membrane and Lactobacillus rhamnosus GG inhibit bacterial attachment to Caco-2 TC7 intestinal cell. Coll. Surf. B 167, 44-53. doi: 10.1016/j.colsurfb.2018.03.044

Hilleringmann, M., Giusti, F., Baudner, B. C., Masignani, V., Covacci, A., Rappuoli, R., et al. (2008). Pneumococcal Pili Are Composed of Protofilaments Exposing Adhesive Clusters of Rrg A. PLoS Pathog 4:e1000026. doi: 10.1371/journal.ppat. 1000026

Hospenthal, M. K., Costa, T. R. D., and Waksman, G. (2017). A comprehensive guide to pilus biogenesis in Gram-negative bacteria. Nat. Rev. Microbiol. 15, 365-379. doi: 10.1038/nrmicro.2017.40.

Kankainen, M., Paulin, L., Tynkkynen, S., von Ossowski, I., Reunanen, J., Partanen, P., et al. (2009). Comparative genomic analysis of Lactobacillus rhamnosus GG reveals pili containing a human- mucus binding protein. Proc. Natl. Acad. Sci. U S A. 106, 17193-17198. doi: 10.1073/pnas.0908876106

Kant, A., Palva, A., von Ossowski, I., and Krishnan, V. (2020). Crystal structure of lactobacillar SpaC reveals an atypical five-domain pilus tip adhesin: Exposing its substrate-binding and assembly in SpaCBA pili. J. Struct. Biol. 211:107571. doi: 10.1016/j.jsb.2020.107571

Kiekens, S., Vandenheuvel, D., Broeckx, G., Claes, I., Allonsius, C., Boeck, I. D., et al. (2019). Impact of spray-drying on the pili of Lactobacillus rhamnosus GG. Microbial. Biotechnol. 12, 849-855. doi: 10.1111/1751-7915. 13426

Krishnan, V. (2015). Pilins in gram-positive bacteria: A structural perspective. IUBMB Life 67, 533-543. doi: 10.1002/iub.1400

Kumar Megta, A., Palva, A., von Ossowski, I., and Krishnan, V. (2019). $\mathrm{SpaB}$, an atypically adhesive basal pilin from the lactobacillar SpaCBA pilus: crystallization and X-ray diffraction analysis. Acta Cryst F 75, 731-737. doi: 10.1107/S2053230X19015358

Laakso, K., Koskenniemi, K., Koponen, J., Kankainen, M., Surakka, A., Salusjärvi, T., et al. (2011). Growth phase-associated changes in the proteome and transcriptome of Lactobacillus rhamnosus GG in industrial-type whey medium. Microbial. Biotechnol. 4, 746-766. doi: 10.1111/j.1751-7915.2011.00275.x

Lavigne, R., Becker, E., Liu, Y., Evrard, B., Lardenois, A., Primig, M., et al. (2012). Direct Iterative Protein Profiling (DIPP) - an Innovative Method for Largescale Protein Detection Applied to Budding Yeast Mitosis. Mole. Cell. Proteom. 11:M111. doi: 10.1074/mcp.M111.012682

Lebeer, S., Claes, I., Tytgat, H. L. P., Verhoeven, T. L. A., Marien, E., von Ossowski, I., et al. (2012). Functional analysis of Lactobacillus rhamnosus GG pili in relation to adhesion and immunomodulatory interactions with intestinal epithelial cells. Appl. Environ. Microbiol. 78, 185-193. doi: 10.1128/ AEM.061926111

McConnell, S. A., Amer, B. R., Muroski, J., Fu, J., Chang, C., Ogorzalek Loo, R. R., et al. (2018). Protein Labeling via a Specific Lysine-Isopeptide Bond Using the Pilin Polymerizing Sortase from Corynebacterium diphtheriae. J. Am. Chem. Soc. 140, 8420-8423. doi: 10.1021/jacs. 8 b05200

Perez-Riverol, Y., Csordas, A., Bai, J., Bernal-Llinares, M., Hewapathirana, S., Kundu, D. J., et al. (2019). The PRIDE database and related tools and resources in 2019: improving support for quantification data. Nucleic Acids Res. 47, D442-D450. doi: 10.1093/nar/gky1106 
Proft, T., and Baker, E. N. (2008). Pili in Gram-negative and Gram-positive bacteria - structure, assembly and their role in disease. Cell. Mol. Life Sci. 66:613. doi: 10.1007/s00018-008-84778474

Rasinkangas, P., Tytgat, H. L. P., Ritari, J., Reunanen, J., Salminen, S., Palva, A., et al. (2020). Characterization of Highly Mucus-Adherent Non-GMO Derivatives of Lacticaseibacillus rhamnosus GG. Front. Bioeng. Biotechnol. 8:1024. doi: 10. 3389/fbioe.2020.01024

Reunanen, J., von Ossowski, I., Hendrickx, A. P. A., Palva, A., and de Vos, W. M. (2012). Characterization of the SpaCBA pilus fibers in the probiotic Lactobacillus rhamnosus GG. Appl. Environ. Microbiol. 78, 2337-2344. doi: 10.1128/AEM.070477011

Suez, J., Zmora, N., Segal, E., and Elinav, E. (2019). The pros, cons, and many unknowns of probiotics. Nat. Med. 25, 716-729. doi: 10.1038/s41591-019-0439-x

Tarazanova, M., Beerthuyzen, M., Siezen, R., Fernandez-Gutierrez, M. M., and de Jong, A, van der Meulen, S. et al. (2016). Plasmid Complement of Lactococcus lactis NCDO712 Reveals a Novel Pilus Gene Cluster. PLoS One 11:e0167970. doi: 10.1371/journal.pone.0167970

Tripathi, P., Beaussart, A., Alsteens, D., Dupres, V., Claes, I., von Ossowski, I., et al. (2013). Adhesion and nanomechanics of pili from the probiotic Lactobacillus rhamnosus GG. ACS Nano 7, 3685-3697. doi: 10.1021/nn400705u

Tripathi, P., Dupres, V., Beaussart, A., Lebeer, S., Claes, I. J. J., Vanderleyden, J., et al. (2012). Deciphering the Nanometer-Scale Organization and Assembly of Lactobacillus rhamnosus GG Pili Using Atomic Force Microscopy. Langmuir 28, 2211-2216. doi: 10.1021/la203834d

Tytgat, H. L. P., Teijlingen, N. H. van Sullan, R. M. A., Douillard, F. P., Rasinkangas, P., Messing, M., (2016). Probiotic Gut Microbiota Isolate Interacts with Dendritic Cells via Glycosylated Heterotrimeric Pili. PLoS One 11:e0151824. doi: 10.1371/journal.pone.0151824 von Ossowski, I. (2017). Novel Molecular Insights about Lactobacillar Sortase-Dependent Piliation. Int. J. Mol. Sci. 18:1551. doi: 10.3390/ijms18071551

von Ossowski, I., Reunanen, J., Satokari, R., Vesterlund, S., Kankainen, M., Huhtinen, H., et al. (2010). Mucosal adhesion properties of the probiotic Lactobacillus rhamnosus GG SpaCBA and SpaFED pilin subunits. Appl. Environ. Microbiol. 76, 2049-2057. doi: 10.1128/AEM.019581959

Wang, F., Cvirkaite-Krupovic, V., Kreutzberger, M. A. B., Su, Z., Oliveira, G. A. P. (2019). An extensively glycosylated archaeal pilus survives extreme conditions. Nat. Microbiol. 4, 1401-1410. doi: 10.1038/s41564-019-0458-x

Zheng, J., Wittouck, S., Salvetti, E., Franz, C. M. A. P., Harris, H. M. B., Mattarelli, P., et al. (2020). A taxonomic note on the genus Lactobacillus: Description of 23 novel genera, emended description of the genus Lactobacillus Beijerinck 1901, and union of Lactobacillaceae and Leuconostocaceae. Int. J. Syst. Evolut. Microbiol. 70, 2782-2858. doi: 10.1099/ijsem.0.004107

Conflict of Interest: The authors declare that the research was conducted in the absence of any commercial or financial relationships that could be construed as a potential conflict of interest.

Copyright (C) 2020 Dos Santos Morais, El-Kirat-Chatel, Burgain, Simard, Barrau, Paris, Borges and Gaiani. This is an open-access article distributed under the terms of the Creative Commons Attribution License (CC BY). The use, distribution or reproduction in other forums is permitted, provided the original author(s) and the copyright owner(s) are credited and that the original publication in this journal is cited, in accordance with accepted academic practice. No use, distribution or reproduction is permitted which does not comply with these terms. 